\title{
DAP kinase and DAP-3: novel positive mediators of apoptosis
}

\author{
Adi Kimchi
}

Programmed cell death is a genetically controlled response for cells to commit suicide. The process, which displays distinctive morphological features, is highly conserved through evolution, and takes place in all nucleated animal cells. It is tightly controlled by environmental stimuli including extracellular diffusible factors, or membrane bound molecules that mediate cell-cell or cell-matrix interactions, and by non-physiological insults to cells such as genotoxic agents. This type of regulation allows the elimination of cells that were either produced in excess during development, have completed their role, are potentially deleterious to the organism, or have become seriously damaged. Programmed cell death is therefore a critical process during embryonic development, tissue remodelling, development of the immune system, and the control of tissue homeostasis. ${ }^{1-3}$ In addition, several disorders associated with the disruption of this fundamental process, have been characterised. While a decrease in the apoptotic rate is linked in some cases to abnormal expansion in cell number (for example, in cancer or autoimmune diseases), an abnormal increase in the apoptotic rate is associated with some cell loss disorders (for example, neurodegenerative diseases). ${ }^{45}$ The link to cancer has been extensively studied from different aspects one of which is the apoptotic checkpoint that safeguards cells against hyperproliferative oncogenic signals. ${ }^{6}$

Several types of programmed cell death have been characterised according to different morphological characteristics. Altogether, the spectrum of morphological hallmarks that has been attributed to the process is very wide, and includes specific nuclear alterations (for example, chromatin condensation followed by its segmentation, internucleosomal DNA fragmentation), cytoplasmic condensation and/or vacuolisation, disruption of cytoskeletal elements, cell surface blebbing, and in some cases, generation of apoptotic bodies that are rapidly phagocytosed by neighbouring cells. ${ }^{7}$ The understanding of molecular mechanisms underlying these different cellular changes has became, in the past few years, an important research goal in the field. The main challenge, in this respect, was to identify the genes that are components of the intracellular pathways that enable cells to receive, process and execute apoptotic signals from their environment. There has been impressive progress in the past few years in understanding the intracellular molecular mechanisms of programmed cell death, emerging from studying both lower invertebrates and mammalian systems (see for example references ${ }^{8-11}$ ). Yet, it is clear that enormous gaps still exist in our knowledge, which means that novel approaches and different insights should be introduced into this field. This paper will focus on one of these recently developed approaches, which is based on a powerful genetic screen applied in mammalian cells. The novel molecular information obtained so far by this approach will be detailed, and future prospects will be discussed.

\section{Development of the technical knock out (TKO) - the method used for the rescue of DAP genes}

The TKO strategy was designed with the intention of establishing a technology that will directly target functionally relevant genes involved in cell death, in a manner similar to the genetic screens used in Drosophila melanogaster or in $\mathrm{C}$ elegans. Instead of mutating the DNA, we directed our efforts at randomly inactivating gene expression by targeting the RNA, thereby bypassing the limitations of mammalian cells that lack the powerful genetic tools available in lower invertebrates. The targeting was performed in an unbiased manner by transfections with anti-sense cDNA expression libraries and producing a wide spectrum of complementary RNAs. ${ }^{12}$ The assumption was that the specific antisense RNA mediated inactivation of a rate limiting death promoting gene would confer some growth advatange to cells that are continuously exposed to a killing cytokine. This advantage could then be used as a powerful forward selection to rescue the relevant cDNA. In other words, the genes of interest are selected and cloned by virtue of a defined phenotypic change-reduced susceptibility to death inducing signals. The cDNA library itself was designed to yield high levels of antisense RNAs by virtue of the fact that:(a) the library was cloned into an EBV-based episomal vector that allows high copy number of episomes per cell, and (b) the expression vector contained an enhancer DNA element that is directly stimulated by the cell death inducing cytokine thus further increasing the expression of antisense RNAs during the selection. ${ }^{12}$ The vector also included a resistance gene to the drug hygromycin B, which allows for survival of only cells that contain the vector, thus eliminating untransfected background cells. In addition, the episomal nature of the vector permitted easier rescue of antisense cDNAs from surviving clones.

Interferon $\gamma(\mathrm{IFN} \gamma)$ was chosen as the preferred external trigger of apoptosis for this purpose. However, the TKO strategy was 


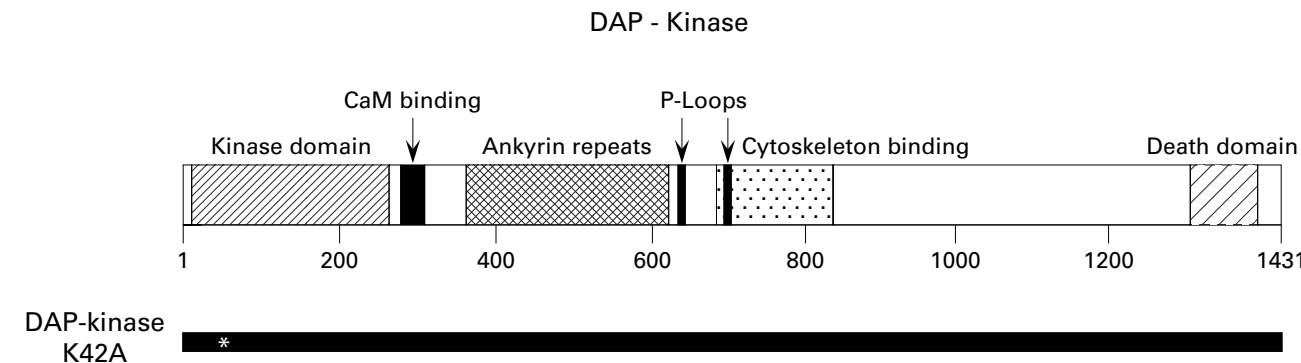

DAP-kinase $1-1271 \Delta \mathrm{DD}$

DAP-kinase $\Delta \mathrm{CaM}$

Figure 1 Schematic representation of DAP-kinase. The various motifs and domains as predicted by the deduced amino acid sequence and/or found by experimental work are shown. Different DAP-kinase mutants used in the work are shown below.

designed in a way that preferentially targets apoptotic genes that belong to the basic machinery of apoptosis, rather than isolating receptor generated early signal transducers. Indeed, in retrospect, after a comprehensive study of the rescued genes, it became clear that most of them mediated the action of different apoptotic triggers including TNF $\alpha$.

After transfection with the anti-sense cDNA library and death induction, surviving cells were isolated, and episomal vectors rescued from these cells were individually tested in a second round of transfection/selection to confirm the phenotype. The plasmids that were scored as positive clones in the second round were further analysed. We named them: Death Associated Protein (DAP) genes, DAP-1 to DAP-5 (DAP-2 was called DAP-kinase). Two groups represented known genes: the thioredoxin gene whose product is involved in redox regulation of intramolecular disulphide bonds, ${ }^{12}$ and cathepsin-D, which is an aspartyl lysosomal protease that turned out to be tightly regulated in apoptosis. ${ }^{13}$ As for the novel DAP genes, the full length sense cDNAs were isolated, specific antibodies were raised against the recombinant proteins, and the expression, function, and biochemical properties of the proteins was studied. ${ }^{14-20}$ Here, we consider in detail one of these rescued DAP genes, DAP-kinase, which was analysed during the past few years from different angles including the possible link of this gene to cancer. ${ }^{21-23}$ In addition, some studies on DAP-3 and cathep$\sin \mathrm{D}$ will be summarised especially in light of their involvement in TNF induced apoptosis.

Initial characterisation of DAP-kinase

The study of this gene provides an interesting paradigm illustrating how from the initial functional selection of an anti-sense cDNA fragment, we proceeded towards the structure/ function analysis of the protein, and then to the notion that a central "death gene", with a potential tumour suppressor activity, has been isolated.

Once the full length cDNA clone of DAP-2 was isolated, the deduced amino acid structure predicted that a novel calmodulin dependent serine/threonine type kinase $(160 \mathrm{kDa})$ had been isolated (see fig 1). Its kinase domain has a classic 11 subdomain composition, typical of serine/threonine kinases, ${ }^{24}$ followed by a region that shares high homology with the calmodulin regulatory domains of other kinases. Adjacent to the latter, we found eight ankyrin repeats followed by two P-loop motifs. ${ }^{14}$ This provided the first reported example of a serine/threonine kinase carrying ankyrin repeats, a motif involved in the formation of stable proteinprotein complexes, which may mediate the interaction of DAP-kinase with its downstream or upstream effectors. Moreover, a typical death domain module was identified at the 3 ' end of the protein, followed by a stretch of amino acids that is rich in serines. ${ }^{15}$ The death domain of DAP-kinase contains all the boxes of homology and the conserved amino acids characteristic of the analogous domains in other death domain cotaining proteins. ${ }^{15}$ Many of these other proteins have a direct involvement in programmed cell death including the p55 TNF receptor, the Fas/APO-1 receptor, DR3-5, FADD/MORT-1, RIP, TRADD, RAIDD and others. ${ }^{25}$ Being a motif composed of several $\alpha$-helical regions, the death domain of DAP-kinase may prompt association with other protein partners critical for the function of this novel type of kinase. The serine rich C-terminal tail of DAP-kinase could negatively regulate a putative function of the death domain, as previously shown for the corresponding region in the Fas/APO- 1 receptor. ${ }^{25}$

Altogether, the predictions made on the basis of the amino acid sequence suggested that a structurally unique kinase had been identified, and emphasised the importance of studying this novel death associated protein both at the biochemical and functional levels.

\section{Biochemistry of DAP-kinase}

A series of biochemical studies confirmed the structural predictions described above. The ability of the protein to phosphorylate itself and an exogenous substrate was shown by in vitro kinase assays. We proved that the recombinant protein was capable of directly binding cal- 
modulin, and that the intrinsic kinase activity was stimulated by $\mathrm{Ca}^{+2} /$ calmodulin. ${ }^{16}$ The removal of the calmodulin regulatory domain ( $\Delta \mathrm{CaM}$ mutant; see fig 1 ) generated a constitutively active kinase (that is, a gain of function mutation). This is consistent with previous information on other well studied calmodulin dependent kinases, in which the calmodulin regulatory domain has inhibitory effects on the kinase activity, relieved by binding to $\mathrm{Ca}^{+2} /$ calmodulin. DAP-kinase activity was abolished by the substitution of a conserved lysine by alanine residue within the kinase domain (K42A), thus generating an inactive kinase mutant with potential dominant negative activity. ${ }^{16}$ Altogether, the complex structure of DAP-kinase that carries multiple functional domains and motifs, place it at an important functional junction within the branched network that leads to cell death, where it may receive and release a variety of input and output signals.

\section{The cellular function of DAP-kinase}

The structural studies of DAP-kinase were then complemented by functional assays. We found that overexpression of wild type DAPkinase reduced the number of viable clones in HeLa cells. ${ }^{16}$ Detailed examination of the cells, early after the transfection (24-48 hours), indicated that overexpression of DAP-kinase killed the cells. The constitutively active $\Delta \mathrm{CaM}$ mutant had stronger death inducing effects in these overexpression assays. In contrast, the catalytically inactive kinase (K42A) completely lost its death inducing activity, and instead, its introduction slightly increased the number and the size of stable clones. The induction of cell death therefore strictly depended on the status of the intrinsic kinase activity, and the catalytic activity of this protein was clearly required for the death promoting function of this protein. These overexpression experiments provided a second independent functional support for DAP-kinase being a death gene, in addition to the anti-sense-RNA mediated protection.

The above mentioned findings concerning the subtle stimulatory effects that K42A DAPkinase mutant exerted on the growth of transfected HeLa clones prompted us to test more directly whether this catalytically inactive form of the kinase could function in a transdominant negative mechanism. We found that transfections with the catalytically inactive K42A mutant protected cells from the IFN $\gamma$ induced cell death. ${ }^{16}$ These studies closed a circle that started by the rescue of a death protective anti-sense cDNA fragment, which interfered with protein expression, and ended by finding that a mutant form of the full length DAP-kinase exerted similar effects, probably by interfering with the function of the endogenous protein.

Additional sets of experiments further strengthened the notion that DAP-kinase is a central cell death gene participating in several apoptotic systems. DAP-kinase gene was found to be widely expressed in many cells and tissues. In addition, we proved that DAP-kinase is involved in cell death induced by $\mathrm{TNF} \alpha$ and by anchorage independent growth. ${ }^{21}$ Also the deletion of the death domain interfered with the death promoting effects of DAP-kinase. As the early signalling cascades generated by p55 TNF receptor or detachment from extracellular matrix do not share any known common elements with IFN $\gamma$ signalling, DAP-kinase probably lies further downstream, and may function as a universal target at which different apoptotic signals eventually converge. Indeed in a recent work we proved that DAP-kinase functions downstream to the DISC (that is, the multi-protein complex formed at the receptor proximal level) and upsteam to some caspases (Cohen et al, submitted data). Thus, the original design of the TKO approach, aimed at targeting elements that function downstream to early signalling, indeed proved to be beneficial.

Immunostaining indicated that DAP-kinase is localised to the cytoskeleton in association with the microfilament system. This was further supported by biochemical fractionations and by application of cytoskeletal disrupting drugs. ${ }^{16}$ Deletion analysis of DAP-kinase mapped the region mediating cytoskeleton binding to a stretch of 200 amino acids localised downstream to the first P-loop motif (see the scheme in fig 1). Most interestingly, cell killing by overexpression of DAP-kinase depended on its correct localisation to the cytoskeleton. This was an important finding as loss of stress fibres and disruption of microfilament organisation occurs at early stages of epithelial and fibroblastic cell death. The specific intracellular localisation of the kinase may therefore provide a mechanistic clue as to how external signals impose these cytoskeletetal changes. ${ }^{16}$

\section{Implications of DAP-kinase in tumorigenesis}

Positive mediators of cell death may be lost or inactivated in tumours and therefore may function as tumour suppressor genes, as clearly documented in the case of p53. Chromosomal localisation studies mapped DAP-kinase to human chromosome 9 band $\mathrm{q} 34.1^{22}$ - a region prone to translocations in human leukaemias and to $\mathrm{LOH}$ in bladder carcinomas. In an attempt to elucidate a role for DAP-kinase in oncogenesis, we first tested the expression of DAP-kinase in human cell lines derived from different neoplasms. DAP-kinase mRNA and protein expression levels were below detection limits in $70 \%$ of B cell lymphoma and leukaemia cell lines and in $30 \%$ of cell lines derived from bladder carcinomas, breast carcinomas, and renal cell carcinomas. ${ }^{23}$ This stood in sharp contrast with the finding that DAP-kinase mRNA was widely expressed in all the tested human and murine tissues, as well as in many immortalised cell lines established from normal cells.

In two bladder carcinoma and in one of the B cell lymphoma cell lines, DAP-kinase expression could be restored by treatment of cells with 5-aza-2'-deoxycytidine, a drug that inhibits DNA methylation. This suggested that loss of expression in these particular cases was attributable to DNA methylation, as previously 
reported for other tumour suppressor genes, such as p16, VHL, and pRB. ${ }^{26}$ Yet, we found, from the tumour cell lines screen, that demetylation is not an exclusive mechanism for suppressing DAP-kinase expression, in accordance with the well established paradigm that tumour suppressor genes may be lost or inactivated by multiple genetic or epigenetic alterations. These experiments provided the first hint that DAP-kinase inactivation may possibly be a causative factor in the formation of tumours, as was further tested in experimental animal model systems.

In parallel, we have carried out a second approach that directly tested in animal models, whether the DAP-kinase gene has tumour suppressor functions. In these experiments, we restored normal expression levels of DAPkinase into tumour cells that have lost it, and assayed the impact of this genetic manipulation on the tumorigenic properties of these cells. This approach became even more attractive once we found that high metastatic lung carcinoma clones, originating from two independent murine lung tumours, lacked DAP-kinase expression, in contrast with their low metastatic counterparts, which expressed normal levels. ${ }^{21}$ FLAG-tagged wild type DAP-kinase was introduced into the high metastatic Lewis carcinoma cells. Stable transfected clones in which DAP-kinase expression was restored to physiological levels displayed normal pattern of cell growth in vitro. These transfectants were therefore assayed for their tumorigenic and metastatic activity in syngeneic mice. Strikingly, restoration of physiological levels of DAP-kinase into the high metastatic Lewis carcinoma cells suppressed their ability to form lung metastases after intravenous injections into mice. The in vivo effects were proportional to the levels of the ectopically expressed DAPkinase, and even the low expressors, containing levels that were below the normal levels, displayed reduced metastatic activity. ${ }^{21}$ The transgene also delayed local tumour growth in a foreign micro environment, yet this feature was less sensitive to DAP-kinase ectopic expression than the metastatic activity.

By selecting in vivo rare lung metastases, after injections of the original low metastatic cells into irradiated syngeneic mice, we found that the developement of these metastatic lesions correlated with loss of the endogenous DAP kinase expression. Moreover, treatment of cells recovered from one of these DAPkinase negative lung lesions with 5-aza-2'deoxycytidine, restored protein expression to the normal levels. DNA methylation was therefore also responsible for silencing the endogenous DAP-kinase gene in some of the in vivo selected lung lesions. ${ }^{21}$ Altogether, these experiments suggested that loss of DAP-kinase expression provides a positive selective advantage during the formation of lung metastases.

Next, the mechanisms underlying the suppressive effects of DAP-kinase on metastasis and local tumour growth were studied. In situ TUNEL staining was performed on histological sections of local tumours. It was found that the apoptotic index in the slow growing local tumors, formed by the DAP-kinase transfected cells, was higher than the value measured in the tumour mass formed by the control clone. ${ }^{21}$ These results provided the first indication implicating the DAP-kinase gene in augmenting the threshold sensitivity of the tumour cells to apoptotic signals. To directly tackle this issue, the transfected cells were exposed in vitro to two types of apoptotic stimuli, of the sort encountered by metastasising cells at the different stages of malignancy, such as $\mathrm{TNF} \alpha$ and anchorage independent cell growth. The transfectants displayed higher sensitivity to the apoptotic effects of $\mathrm{TNF} \alpha$ : fragmented nuclei appeared much faster, and total cell death was greater. A second type of an apoptotic stress was induced by growing cells under anchorage independent conditions in soft agar. In contrast with the parental high metastatic clone, which formed large viable colonies in semi-solid medium, the various DAP-kinase transfected clones formed small colonies comprising of apoptotic cells. Based on these experiments we suggested that DAP-kinase mediated suppression of metastasis results, at least in part, from increased sensitivity to various death inducing stimuli. We propose that loss of DAP-kinase expression provides a novel mechanism that links suppression of apoptosis to metastasis. ${ }^{21}$

\section{DAP-3}

The DAP-3 gene, localised to chromosome 1 band $\mathrm{q} 21,{ }^{18}$ codes for a protein of $46 \mathrm{kDa}$ that carries a potential "P-loop" motif, suggesting that it is a nucleotide binding protein. The gene is transcribed into a single $1.7 \mathrm{~kb}$ mRNA, which is ubiquitously expressed in different cells and tissues. ${ }^{17}$ The notion that DAP-3 functions as a major positive mediator of cell death was supported by two independent lines of evidence. One of them is based on the original assay, which in fact led to the identification of this gene-that is, protection from cell death conferred by reduced expression of the protein. The assay in this experiment was based on neutral dye uptake into viable cells. It showed that transfection with a vector expressing antisense DAP-3 RNA caused a significant increase in the fraction of cells that remained viable in the continuous presence of IFN $\gamma$. Western blot analysis, performed by using antiDAP-3 polyclonal antibodies, confirmed that the anti-sense RNA expression indeed reduced the steady state levels of DAP-3 protein in the continuous presence of IFN $\gamma .{ }^{17}$

The second line of evidence came from overexpression of the full length sense cDNA. We found in this respect that ectopic expression of DAP-3 from a constitutive promoter induced the death of a variety of cell lines and primary cell cultures. ${ }^{20} \mathrm{~A}$ comprehensive study of a series of deletion mutants resulted in the characterisation of a truncated DAP-3 protein, named $\Delta 230$, which acted as a dominant negative mutant. Its inhibitory function on DAP-3 depended on the status of the P-loop motif. Ectopic expression of $\Delta 230$ DAP-3 mutant protected cells from TNF induced apoptosis, in a protein synthesis independent manner, suggesting that DAP-3 is required for mediat- 
ing this process. ${ }^{20} \mathrm{By}$ immunostaining analysis it was shown that the endogenous DAP-3 protein is localised to cellular vesicles the nature of which is currently being studied.

\section{The cathepsin D protease connection to programmed cell death}

One of the surprises in the TKO selection was the identification of an anti-sense cDNA fragment directed against human cathepsin D aspartic protease. Obviously, proteases were expected to be isolated during the selection, yet our prediction was that they will fall into the known family of caspases. The lysosomal cathepsin $\mathrm{D}$ has a wide range of activities in mammalian cells, mostly executed in the lysosomal compartment during steady state growth conditions. It was only after a detailed study of the fate of this protease during cell death, that an interesting unexpected scenario has emerged, consistent with an active role in apoptosis. ${ }^{13}$

The initial analysis of the secondary stable clones indicated that the high levels of cathep$\sin \mathrm{D}$ anti-sense RNA protected the HeLa cells from IFN $\gamma$ and Fas/APO-1 induced death. As pepstatin A, a peptide inhibitor of aspartic proteases, was extensively used in the past to specifically suppress cathepsin D activity in intact cells, we used it in our systems. It was found that this peptide inhibitor suppressed cell death in HeLa cells exposed to IFN $\gamma$ and to the antiFas/APO-1 agonistic antibodies. Protection by pepstatin A was also detected during the TNF $\alpha$ induced programmed cell death of U937 cells. ${ }^{13}$ We thus established the role of this protease in three different apoptotic systems.

The next challenge was to understand how a house keeping lysosomal protease is recruited to the tighly regulated process of programmed cell death. We found that this gene has an interesting pattern of regulation during cell death. The mRNA expression of cathepsin D was increased and the processing of the protein was regulated in the dying cells. As a consequence, high steady state levels of an intermediate, proteolytically active, single chain form of this protease accumulated in cells. ${ }^{13}$ This form is present at low steady state levels in normally growing cells, and is usually found in pre-lysosomal compartments. Its abnormal accumulation in apoptotic cells further suggested that changes in cathepsin $\mathrm{D}$ intracellular localisation, and hence in the spectrum of potential substrates might occur during cell death.

Recently, Wu and coworkers reported that cathepsin D has a role in p53 dependent apoptosis. Using subtractive hybridisation screening, they isolated cathepsin D as an upregulated transcript appearing in cells udergoing adriamycin induced apoptosis, in a p53 dependent manner. ${ }^{27}$ They found that cathepsin D mRNA levels increased in wild type p53 expressing cell lines after adriamycin addition. Other DNA damaging reagents were similarly effective in increasing cathepsin D expression. Two p53 DNA binding sites were identified in the cathepsin D promoter. They bound to $\mathrm{p} 53$ in vitro, and seemed to mediate transactivation by $\mathrm{p} 53$ of a reporter gene driven by the cathepsin D promoter. It seems from this work that cathepsin D also mediates apoptotic signals that involve DNA damage and are p53 dependent, thus further extending the spectrum of apoptotic signals that use this aspartic protease to intracellular signals as well.

Altogether, cathepsin D may be added to the growing list of proteases that function as positive mediators of apoptosis. How its function is related to the cascade of caspase activation during apoptosis is an issue to be studied.

\section{Discussion}

While the research on the novel DAP genes is still very far from completion, a few common interesting paradigms already emerge. The start points in the isolation of these genes were the selected short cDNA fragments, which in most cases directed the transcription of high levels of anti-sense RNAs. Later on, once the full length proteins were further characterised, we designed specific mutations within the DAP genes, or used small peptide inhibitors, to illustrate by a few independent approaches the rate limiting functions that these genes display in apoptosis. Two classic examples are the K42A dominant negative mutant of DAP-kinase and the pepstatin A peptide inhibitor, which suppressed the function of DAP-kinase and of cathepsin D proteins, respectively, and thereby protected cells from apoptosis.

DAP-kinase and DAP-3 are highly conserved among different species. Recently we have isolated the $\mathrm{C}$ elegans homologues of DAP-kinase and DAP-3, displaying $40 \%$ and $37 \%$ identity at the amino acid level (Kissel et $a l^{20}$ and unpublished results). The ubiquitous expression of these genes in many cell types and tissues, and the high degree of conservation among different organisms imply that we managed to isolate evolutionary conserved and important components of cell death pathways that are common to various cells. Moreover, we found that the function of these genes is not restricted to a single external apoptotic stimulus. DAP-kinase, DAP-3, and cathepsin D also mediate Fas/APO-1 and TNF $\alpha$ induced cell death. Most interestingly, we recently found that restoration of DAP-kinase into DAPkinase negative cells also conferred susceptibility to death that is induced by detachment from extracellular matrix (named anoikis). ${ }^{21}$ Finally, the recent study by Wu and coworkers showing that cathepsin D is directly transactivated by p53, and is required for transmitting the apoptotic effects of wild type p53 in response to DNA damaging drugs, ${ }^{27}$ clearly proves that this protease also mediates intracellular apoptotic signals. Altogether, the spectrum of apoptotoc stimuli that converge into DAP-genes and cathepsin D is very wide, suggesting that the original design of the pTKO-1 vector, which was set for cloning downstream common apoptotic elements ${ }^{12}$ indeed proved itself.

Regulation of DAP genes by the apoptotic signals is another issue that deserves close attention. If a gene that was initially rescued through its rate limiting function during cell death, becomes modified by the apoptotic sig- 
nal directly or indirectly (at the transcriptional/ post-transcriptional/post-translational levels), then it can undoubtedly be considered as an intrinsic part of an apoptotic pathway. The best example obtained so far is the pattern of cathepsin D regulation during cell death that consists of a few independent levels. Firstly, the above mentioned killing cytokines increased the cathepsin D mRNA expression, probably in a p53 independent manner (as HeLa cells carry the E6 viral oncogene). ${ }^{13}$ Secondly, the p53 binding sites in the promoter region reflected another layer of regulation during cell death. ${ }^{27}$ And thirdly, the killing cytokines imposed a specific block in the processing of this protein, resulting in the accumulation of high levels of a single chain precursor that is proteolytically active. ${ }^{13}$

Finally, the link to cancer and to other disorders is an important issue in evaluating the physiological role of DAP genes. The best indications that we have so far relate to the DAP-kinase gene that possesses strong antimetastatic activities. This aspect in the function of the gene was attributed, at least partially, to the high susceptibility that it conferred to apoptotic stimuli, some of which operate during metastasis. ${ }^{21}$ For instance, upon entering into circulation, the invading metastasising cells may be killed by interactions with monocytes, natural killer cells, and neutrophils, by exposure to cytokines, to nitric oxide anions, or by different mechanical stresses. The loss of DAP-kinase conveys to the metastasising cells strong positive selection to resist the apoptotic stress, as also shown by the in vivo selections of lung metastases in mice model systems. ${ }^{21}$ Frequent inactivating mutations in human tumours, if detected, should provide an additional independent support that DAP genes display an important function in keeping cells under the tight control of apoptotic mechanisms that take place normally in vivo. Obviously, other mechanisms may contribute to the tumour suppressive functions of DAP genes, in addition to mediating apoptotic responses.
One of the major challenge now is to analyse how these diverse activities constitute biochemical pathway(s) leading to programmed cell death. This depends on studying the nature of interactions between the individual DAP genes on the one hand, and determining their functional position with respect to the other well known positive and negative mediators of apoptosis, on the other hand. For instance, the possible functional interactions with members of the bcl-2 family, or with the caspases, can be assessed now by stable or transient transfections, or by the use of specific peptide inhibitors. Caspases could function in parallel to the biochemical pathways that involve DAP genes, or alternatively may be integrated into these pathways either as upstream modulators, downstream executors, or both.

1 Wyllie AH, Kerr JF, Currie AR. Int Rev Cytol 1980;68:251306.

2 Raff MC. Nature 1992;356:397-400

3 Schwartz LM, Osborne BA. Immunol Today 1992;14:58290 .

4 Thompson CB. Science 1995;267:1456-62.

5 Barinaga M. Science 1998;281:1302-4.

6 Evan G, Littlewood T. Science 1998;281:1317-22.

7 Clarke PG. Anat Embryol Berl 1990;181:195-213.

8 Hengartner MO, Horvitz HR. Curr Opin Gene Dev 1994;4:581-6.

9 Steller H. Science 1995;267:1445-9.

9 Steller H. Science 1995;267:1445-9.

11 Adams JM, Cory S. Science 1998;281:1322-5.

12 Deiss LP, Kimchi A. Science 1991;252:117-20.

13 Deiss LP, Galinka H, Berissi H, et al. EMBO J 1996;15: 3861-70.

4 Deiss LP, Feinstein E, Berissi H, et al. Genes Dev 1995;9:15-30

15 Feinstein E, Wallach D, Boldin M, et al. Trends Biochem Sci 1995;20:342-4

16 Cohen O, Feinstein E, Kimchi A. EMBO J 1997;16:998-

17 Kissil J, Deiss LP, Bayewitch M, et al. J Biol Chem 1995;270:27932-6.

18 Kissil J, Kimchi A. Cytogenet Cell Genet 1997;77:252.

19 Levy-Strumpf N, Deiss LP, Berissi H, et al. Mol Cell Biol 1997; 17:1615-25.

20 Kissil J, Cohen O, Raveh T, et al. EMBO J 1999;18:353-62.

21 Inbal B, Cohen O, Polak-Charcon S, et al. Nature Inbal B, Cohen
1997;390:180-4.

22 Feinstein E, Druck T, Kastury K, et al. Genomics 1995;29: 305-7.

23 Kissil J, Feinstein E, Cohen O, et al. Oncogene 1997;15: 403-7.

4 Shoemaker MO, Lau W, Shattuck RL, et al. J Cell Biol 1990;111:1107-25.

25 Ashkenazi A, Dixit VM. Science 1998;281:1305-8. 26 Laird PW, Jaenisch R. Annu Rev Genet 1996;30:441-64. 27 Wu GS, Saftig P, Peters C, et al. Oncogene 1998;16:217783 . 